\title{
STRING THEORY LANDSCAPE AND THE STANDARD MODEL OF PARTICLE PHYSICS*
}

\author{
DIETER LÜST \\ Arnold-Sommerfeld-Center for Theoretical Physics \\ Department für Physik, Ludwig-Maximilians-Universität München \\ Theresienstraße 37, 80333 München, Germany \\ and \\ Max-Planck-Institut für Physik \\ Föhringer Ring 6, 80805 München, Germany \\ luest@theorie.physik.uni-muenchen.de,luest@mppmu.mpg.de
}

\begin{abstract}
In this paper we describe ideas about the string landscape, and how to relate it to the physics of the Standard Model of particle physics. First, we give a short status report about heterotic string compactifications. Then we focus on the statistics of D-brane models, on the problem of moduli stabilization, and finally on some attempts to derive a probability wave function in moduli space, which goes beyond the purely statistical count of string vacua.
\end{abstract}

\section{Introduction}

String theory is a subject with beautiful connections to mathematics, in particular to differential and algebraic geometry. Moreover, it is well known that string theory provides a consistent formulation of quantum gravity, and one of its nicest achievements is the microscopic count of black hole entropies in string theory that precisely reproduces the thermodynamic area law of Bekenstein and Hawking including corrections from higher derivative gravity couplings ( $\mathrm{se}^{1}$ for a recent review). However not only gravity is an automatic outcome of string interactions, but also gauge interactions follow from the string world sheet formulation. In addition, there exist a deep relation between gauge and gravitational interactions in string theory, which manifests itself in the form of the AdS/CFT correspondence, ${ }^{2-4}$ namely the duality between quantum gravity in 5-dimensional anti-De Sitter space $\left(A d S_{5}\right)$ and conformal gauge theories in four-dimensions on the boundary of $A d S_{5}$ (the holographic principle).

However, still one of the most important issues is how to relate string theory to the observables in the low energy physics world. Specifically, since we know that string theory provides an unification of all interactions and particles, we want to know how the Standard Model (SM) of elementary particles with gauge group $G=S U(3) \times S U(2) \times U(1)_{Y}$ and with 3 generations of quark and leptons follows from string theory. Next, string theory should provide a framework for computing all couplings of the SM. Space-time supersymmetry is automatically built into string theory; hence we have to find out how supersymmetry gets broken in string theory,

${ }^{*}$ Contribution to the 11th. Marcel Grossmann Meeting on General Relativity in Berlin, July 2006 
and what are the consequences of a possible low energy supersymmetry breakdown for the physics at the LHC. In fact, it is one of the most interesting problems in string phenomenology to provide a translation between the low energy effective string action and the chain of data, which is expected from the LHC. Finally, it would be of course most desirable for string theory to provide new testable experimental signatures, like extra dimensions or the discovery of microscopic black holes and their Hawking radiation, supporting in this way string theory (or providing arguments for abandoning it).

One possible approach to string theory is the top-down approach, which starts from the unification of gravity and gauge interactions at very high energies, and then tries to deduce all low energy observables from investigating the mathematical structures of the theory. Although we do not yet know the typical string scale $M_{\text {string, }}$, where the unification of gravity and gauge interaction takes places, one often assumes, at least from a conservative point of view, that this happens at the Planck scale of about $M_{\text {Planck }} \simeq 10^{19} \mathrm{GeV}$ (there are also alternatives models with much lower string scale ${ }^{5}$ ). However no direct experiments will guide us through the physics at such high energies, a fact which makes the top-down approach very troublesome. Another problem for the top-down approach is the fast proliferation of string solutions, the so-called string landscape problem, which we will describe in the following. The string landscape is defined to be the space of all possible solutions of the string equations of motion. In ten space-time dimensions, there exist just five different formulations of string theory (two heterotic strings, type I type IIA and type IIB superstrings). Exploring several kind of duality symmetries, it is conjectured that all these string theories can be unified into M-theory, where also 11-dimensional supergravity is included. However the number of lower-dimensional string solutions, i.e. lower dimensional string groundstates, which are obtained after compactification, is enormous. Already in 1984, within the covariant lattice construction, ${ }^{6}$ the number of possible four-dimensional string ground states was estimated to be of order $10^{1500}$. More recently, the number of discrete flux vacua on a generic Calabi-Yau manifold was shown to be of order $10^{500}$. $^{7,8}$ Taken seriously, this vast landscape of distinct string vacua really implies a big question mark concerning the predictivity of string theory, since each point in the landscape essentially corresponds to a different universe with different particle physics and cosmological properties. To deal with such a huge number of possibilities, certain strategies are required in order to proceed within the top-down approach. One possible and legitimate approach is given by the investigation of the statistical properties of the string landscape. I.e. one has to determine by statistical methods what is the fraction of string vacua with good phenomenological properties. Possible statistical correlations resp. anti-correlations would be especially worth to be discovered, like e.g. between the number of families and the rank of the low-energy gauge groups, because they could provide a step towards verifying or resp. falsifying string theory. In addition, it was argued that the landscape could be the clue for statistically explaining the hierarchy problem in physics, and especially the smallness of the observed cosmological constant. Namely, 
the tiny observed value of $\Lambda / M_{\text {Planck }}^{4} \simeq 10^{-120}$ could be statistically explained ${ }^{9}$ if the number of vacua is of order of $10^{120}$, which indeed seems to be the case in string theory. Eventually, the statistical approach is likely to be merged with the anthropic principle $^{10}$ (see also ${ }^{11}$ ). Concerning the evolution of the universe (see e.g. ${ }^{12}$ ), the anthropic principle essentially requires a multiverse with a huge number of bubbles, with each being filled by one of the vacua of the landscape. The population of all possible bubbles in the universe is possible in the context of eternal inflation, where transitions between different bubbles due to quantum tunneling processes are going to happen.

Another useful insight into the landscape discussion might be provided by the quantum mechanical wave function (Hartle-Hawking wave function ${ }^{13}$ ) of the universe, which describes a probability measure, namely the likelihood for each point in the landscape to be populated. In string theory, it is natural to assume that this probability measure is linked to an entropy functional in the landscape, i.e. the number of microscopic string excitations that correspond to each ground state. Clearly, this point of view is reminiscent to the discussion of black hole entropies, where the macroscopic black hole entropy can be explained by counting the corresponding microscopic string degrees of freedom. We will come to an explicit proposal how to relate black hole entropies to probability functions in the flux landscape at the end of this paper.

Complementary to the top-down efforts, the bottom-up approach is very important for connecting string theory with the real world. Here one tries to build consistent string models which contain as many SM features as possible. First one tries to build string models that contain as massless states the particles of the SM, gauge bosons and three families of quarks and leptons. Next, one has to derive the low-energy effectice action of the massless fields, in order to compute their couplings, like gauge couplings and Yukawa couplings, which eventually can be compared with the experimentally known values. Here another problem has to be solved, namely the problem of moduli stabilization. String compactifications generically contain several massless moduli fields with flat potential, which correspond to geometrical or other parameters of the internal space. These have to be fixed, since the lowenergy couplings of the massless fields are functions of the moduli. In order to make predictions one has to know the values of the moduli. In addition, massless moduli would overclose the universe and also cause un-observed new forces. ${ }^{14}$

One additional guiding principle for string model building is the fact that, although the energy scale of the SM is around $100 \mathrm{GeV}$, the SM is not a complete model but certainly requires new physics at mass scales much higher than $100 \mathrm{GeV}$. In fact it is encouraging that several puzzles of the SM, most notably the hierarchy problem together with the problem of the missing dark energy, can be explained in one stroke, namely by low-energy space-time supersymmetry around the corners at about $1-10 \mathrm{TeV}$. This fact gave rise to the formulation of the minimal supersymmetric standard model (MSSM) or some variants thereof. Since supersymmetry is built into string theory from the very beginning, the discovery of low energy su- 
persymmetry would be also an additional argument in favor of string theory. The smallness of neutrino masses can be nicely explained by the see-saw mechanism, pointing to another new energy scale between $10^{10}-10^{12} \mathrm{GeV}$. Finally, the unification of gauge coupling constants of the SM happens at the GUT scale of $10^{16} \mathrm{GeV}$, and gauge coupling apparently works best within the MSSM. Therefore neutrino masses and gauge coupling unification should be another ingredient for string model building.

Another window into new physics beyond the SM comes from astrophysics and cosmology. Beautiful experiments, most notably COBE and WMAP, provided a precise image of cosmic microwave background radiation including its small density variations. In this way the inflationary scenario of the early universe is now established as the standard model for cosmology. In addition, we know from the astrophysical measurements that our universe is spatially flat. Its energy density is dominated to about $74 \%$ by a dark energy component, which behaves very similarly to a positive cosmological constant. The explanation of this mysterious dark energy is one of the biggest challenges for astroparticle physics, and hence also for string theory. The remaining $26 \%$ of the energy density is split into so far directly undiscovered dark matter particles (WIMPS), which account for $22 \%$ of the total energy density, and into a left-over $4 \%$ component of visible SM matter fields. Many properties of the dark matter fields are still unknown, although one very promising candidate for dark matter is the lightest supersymmetric particle (LSP) in the MSSM. So, strings also should be helpful to identify the nature of dark matter.

In summary, successful string model building must take into account all these phenomenological boundary conditions coming from the SM, from particle physics beyond the SM and also from cosmology. The top-down constructions which start from the geometry of the compactification space must go hand in hand with the bottom-up approach, where one is guided by the phenomenological data. In this way, a (not necessarily one-to-one) map between geometrical and topological properties of the compactifications spaces and the particle physics observables will be provided. This dictionary between geometry/topology and particle physics/cosmology is one of the most interesting aspects of string theory, and will be demonstrated in this paper by several examples.

The outline of the paper is the following: in the next section we discuss heterotic string compactifications, and especially new vector bundle constructions which are helpful in order to derive the spectrum and the particle content of the MSSM. In section three type IIA intersecting D-brane models will be reviewed. We will address the statistical properties of these models, i.e. we discuss the question, how many consistent D6-brane embeddings on a given closed string background exist in total, and what is the fraction of them that come close the MSSM. Next we will consider the problem of moduli stabilization and also of supersymmetry breaking by adding background fluxes and taking into account effects from non-perturbative string instantons. Finally, in section four, we will discuss some aspects about the relation between flux vacua and black hole entropies, and the related problem how 
to formulate for flux compactificatons a wave function in moduli space.

\section{Heterotic string compactifications}

In ten dimensions there exist two heterotic strings models with $\mathcal{N}=1$ supersymmetry (16 supercharges) and gauge groups, $G_{10}=E_{8} \times E_{8}, S O(32)$. Compactifications of the heterotic string that preserves $\mathcal{N}=1$ supersymmetry in four dimensions ( 4 supercharges) are built following essentially two steps:

- A suitable choice of an internal, six dimensional manifold $\mathcal{M}_{6}$, which can be a Calabi-Yau (CY) manifold, a supersymmetric orbifold, or, in more complicated cases with background fluxes, a space with $S U(3)$ group structure. We will focus on Calabi-Yau compactifications.

- A suitable choice of a stable vector bundle $V$ with structure group $H$ over $\mathcal{M}_{6}$. In order to preserve space-time supersymmetry, $V$ must be holomorphic, its fields strength has to satisfy

$$
F_{a b}=F_{\bar{a} \bar{b}}=g^{a \bar{b}} F_{a \bar{b}}=0,
$$

where $g^{a \bar{b}}$ is the inverse metric of the complex space $\mathcal{M}_{6}$. Furthermore one has to demand the absence of tadpoles, i.e. $d H_{3}=0$, with $H_{3}$ being the NS 3 -form field strength. This implies the vanishing of the second Chern class of $V$ :

$$
c_{2}(V)=c_{2}(T),
$$

where $c_{2}(T)$ is the second Chern class of the tangent bundle of the CYmanifold. The observed gauge group $G_{4}$ in four dimensions is given by the commutant of $H$ in $G_{10}$ :

$$
G_{10} \supset H \times G_{4} .
$$

Finally, the number of chiral, massless matter fields (in a given representation of $G_{4}$ - see later for more details) is determined by the third Chern number of $V$ :

$$
N_{F}=\frac{1}{2} c_{3}(V) .
$$

Now there exists essentially two classes of heterotic bundle constructions in the literature:

(i) $H$ being a simple Lie-group:

These bundles were explicitly constructed by a number of groups, ${ }^{15-25}$ where most of the explicit constructions use as a CY base space an elliptically fibred Calabi-Yau manifold. Since $H$ is simple, the four-dimensional gauge group is simple, too. Hence this construction is very suitable for construction GUT models in four dimensions. 
E.g.:

$$
\begin{aligned}
& H=S U(3) \quad \longrightarrow \quad G_{4}=E_{6}, \\
& H=S U(4) \quad \longrightarrow \quad G_{4}=S O(10), \\
& H=S U(5) \quad \longrightarrow \quad G_{4}=S U(5) .
\end{aligned}
$$

It is a generic feature of these vector bundles, that the four-dimensional spectrum does not contain Higgs fields in the adjoint representation of $G_{4}$. Therefore, in order to further break these GUT groups to the gauge group of the SM, one needs additional discrete Wilson lines. In fact, following this method, three generation models without exotic particles can be constructed.

(ii) $H$ being a non-simple Lie-group:

Here, the structure group $H$ contains at least one Abelian $U(1)$ factor, i.e. it is of the form: $H=H^{\prime} \times U(1) .{ }^{26-29}$ It follows that also $G_{4}$ contains the same $U(1)$ gauge factor:

$$
G_{4}=G_{4}^{\prime} \times U(1) .
$$

Of course, this feature is required, if one likes to construct heterotic CY compacatifications, which directly lead to the SM gauge group, or other models with $U(1)$ factors, like flipped $S U(5)$ GUT's with gauge group $G_{4}=S U(5) \times U(1)$. Explicit models can be again constructed on elliptically fibred CY-spaces. A detailed analysis of the topology of these vector bundles reveals the following interesting relation between the Euler numbers $\chi$ of $V$ and the number of SM particle representations in four dimensions: ${ }^{28}$

\begin{tabular}{|c||c|c|}
\hline \hline$S U(3) \times S U(2) \times U(1)_{Y} \times E_{7}$ & bundle & SM part. \\
\hline \hline$(\mathbf{3}, \mathbf{2}, \mathbf{1})_{\frac{1}{3}}$ & $\chi(V)=g$ & $q_{L}$ \\
$(\mathbf{3}, \mathbf{2}, \mathbf{1})_{-\frac{5}{3}}$ & $\chi\left(L^{-1}\right)=0$ & - \\
\hline$(\overline{\mathbf{3}}, \mathbf{1}, \mathbf{1})_{\frac{2}{3}}$ & $\chi\left(\bigwedge^{2} V\right)=g$ & $d_{R}^{c}$ \\
$(\overline{\mathbf{3}}, \mathbf{1}, \mathbf{1})_{-\frac{4}{3}}$ & $\chi\left(V \otimes L^{-1}\right)=g$ & $u_{R}^{c}$ \\
\hline$(\mathbf{1}, \mathbf{2}, \mathbf{1})_{-1}$ & $\chi\left(\bigwedge^{2} V \otimes L^{-1}\right)=g$ & $l_{L}$ \\
$(\mathbf{1}, \mathbf{1}, \mathbf{1})_{2}$ & $\chi(V \otimes L)+\chi\left(L^{-2}\right)=g$ & $e_{R}^{c}$ \\
$(\mathbf{1}, \mathbf{1}, \mathbf{5 6})_{1}$ & $\chi\left(L^{-1}\right)=0$ & - \\
\hline
\end{tabular}

Various concrete models leading to $\chi(V)=g=3$ were constructed in. ${ }^{28}$ Note that in these heterotic compactifications with Abelian vector bundles there can be more than one $U(1)$ gauge group factors that become massive due to the coupling 
to pseudo scalar moduli fields. This is analog to type II orientifolds, which we will discuss in the next section. In fact, heterotic compactifications with Abelian vector bundle are expected to be the string dual of type I compactifications with magnetized D9-branes.

Let us close the short overview over new heterotic compactifications with a few more remarks.

- Gauge coupling unification of the SM gauge coupling constants typically occurs in perturbative heterotic compactifications at a scale of order $M_{G U T} \simeq g_{\text {string }} M_{\text {string }} \simeq 10^{17} \mathrm{GeV}$. This is about one order of magnitude too high compared with the phenomenologically preferred GUT-scale. However this constraint can be relaxed by taking into account moduli dependent, perturbative gauge threshold corrections, ${ }^{30-33}$ or including nonperturbative corrections in heterotic M-theory. ${ }^{34,35}$ Other alternatives like models with non-standard hypercharge embeddings are summarized in. ${ }^{36}$

- Heterotic moduli stabilization, including all vector bundle moduli, is still a quite difficult problem. For some attempts of heterotic flux compactifications see, ${ }^{37-41}$ and for the the issue of stabilizing moduli in heterotic M-theory consult. ${ }^{42}$

- Recently, phenomenologically very attractive heterotic models have been constructed on orbifold spaces ${ }^{43-45}$ and also using free fermions, ${ }^{46-48}$ and aspects of the corresponding heterotic landscape were discussed in. ${ }^{49-51}$

\section{Intersecting D-brane models}

Now let us turn to type II orientifold compactifications to four-dimensions on sixdimensional manifolds $\mathcal{M}_{6}$, which we first discussed in. ${ }^{52-56}$ In order to incorporate non-Abelian gauge interactions and to obtain massless fermions in non-trivial gauge representations, one has to introduce D-branes in type II superstrings. Specifically there exist three classes of four-dimensional models:

(i) Type I compactifications with D9/D5 branes:

This class of IIB models contain different stacks of D9-branes, which wrap the entire space $\mathcal{M}_{6}$, and which also possess open string, magnetic, Abelian gauge fields $F_{a b}$ on their world volumes (magnetized branes). In other words, $F_{a b}$ corresponds to open string vector bundles, and this class of models is string dual to heterotic string compactifications. For reasoning of Ramond tadpole cancellation, one also needs an orientifold 9-plane (O9-plane). In addition one can also include D5-branes and corresponding O5-planes. In the heterotic dual description the D5/O5 open strings correspond to the non-perturbative sector of the theory. Since the open string gauge fields $F_{a b}$ induce mixed boundary conditions on the D-branes, the internal compact space can be regarded as a non-commutative space.

(ii) Type IIB compactifications with D7/D3 branes: 
Here we are dealing with different stacks of D7-branes, which wrap different internal 4-cycles, which intersect each other. The D7-branes can also carry non-vanishing open string gauge flux $F_{a b}$. In addition, one can also allow for D3-branes, which are located at different point of $\mathcal{M}_{6}$. In order to cancel all Ramond tadpoles one needs in general O3- and O7-planes.

(iii) Type IIA compactifications with D6 branes:

This class of models contains intersecting D6-branes, which are wrapped around 3-cycles of $\mathcal{M}_{6}$. Now, orientifold O6-planes are needed for Ramond tadpole cancellationa

D-brane models of these three different classes generically can be mapped onto each other by T-duality, resp. IIA/IIB mirror symmetry including open strings and Dbranes, and therefore are essentially on equal footing. Hence, in the following we will concentrate on IIA intersecting D6-brane models (class (iii)). Since we want to engineer the SM, it turns out that one needs at least four stacks of D6-branes: ${ }^{58,59}$

\begin{tabular}{|c|c|c|c|}
\hline Stack a: & $N_{a}=3$ & $S U(3)_{a} \times U(1)_{a}$ & QCD branes \\
\hline Stack b: & $N_{b}=2$ & $S U(2)_{b} \times U(1)_{b}$ & weak branes \\
\hline Stack c: & $N_{c}=1$ & $U(1)_{c}$ & right brane \\
\hline Stack d: & $N_{d}=1$ & $U(1)_{d}$ & leptonic brane \\
\hline
\end{tabular}

Here, $N_{a}$ denotes the number of D6-branes in each stack. The intersection pattern of the four stacks of D6-branes can be depicted in the next figure:

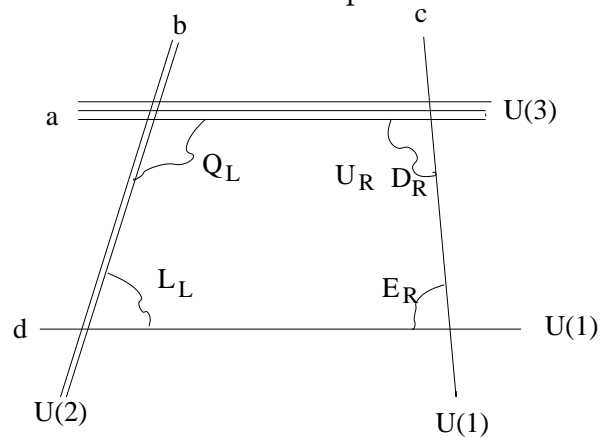

This local stack of SM intersecting D6-branes has to be embedded into the compact space $\mathcal{M}_{6}$. For reasons of Ramond tadpole conditions one usually needs more D-branes than this SM brane configuration. These additional D-branes build the socalled hidden gauge sector. This scenario can be depicted in the following picture of the internal space:

also coisotropic D8-branes ${ }^{57}$ can be in principle included, which we however leave out from the discussion. 


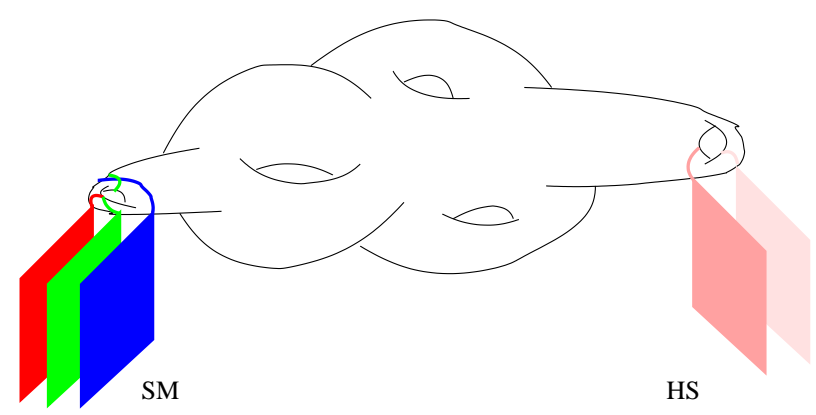

Many details on the construction of open string intersecting brane models and how to relate the to the SM can be found in various review articles. ${ }^{60-65}$ Let us therefore just summarize the main aspects of the intersecting D6-brane models.

- We assume that six spatial directions are described by a compact space $\mathcal{M}_{6}$. In addition, a consistent orientifold projection is performed. This yields O6-planes and in general changes the geometry. The bulk space-time supersymmetry is reduced to $\mathcal{N}=1$ by the orientifold projection. To be more specific we will consider a type IIA orientifold background of the form

$$
\mathcal{M}^{10}=\left(\mathbb{R}^{3,1} \times \mathcal{M}_{6}\right) /(\Omega \bar{\sigma}), \quad \Omega \text { : world sheet parity }
$$

Here $\mathcal{M}_{6}$ is a Calabi-Yau 3 -fold with a symmetry under $\bar{\sigma}$, the complex conjugation

$$
\bar{\sigma}: z_{i} \mapsto \bar{z}_{i}, i=1, \ldots, 3,
$$

in local coordinates $z_{i}=x^{i}+i y^{i}$. It is combined with the world sheet parity $\Omega$ to form the orientifold projection $\Omega \bar{\sigma}$. This operation is actually a symmetry of the type IIA string on $\mathcal{M}_{6}$. Orientifold 6-planes are defined as the fixed locus

$$
\mathbb{R}^{3,1} \times \operatorname{Fix}(\bar{\sigma})=\mathbb{R}^{3,1} \times \pi_{O 6},
$$

where $\operatorname{Fix}(\bar{\sigma})$ is a supersymmetric (sLag) 3 -cycle on $\mathcal{M}^{6}$, denoted by $\pi_{O 6}$. It is special Lagrangian (sLag) and calibrated with respect to the real part of the holomorphic 3-form $\Omega_{3}$.

Next we introduce D6-branes with world-volume

$$
\mathbb{R}^{3,1} \times \pi_{a},
$$

i.e. they are wrapped around the supersymmetric (sLag) 3-cycles $\pi_{a}$ and their $\Omega \bar{\sigma}$ images $\pi_{a}^{\prime}$ of $\mathcal{M}_{6}$, which intersect in $\mathcal{M}_{6}$. Since the D-branes will be wrapped around compact cycles of the internal space, multiple intersections will now be possible. The chiral massless spectrum indeed is completely fixed by the topological intersection numbers $I$ of the 3 -cycles of the configuration. 


\begin{tabular}{lll} 
Sector & Rep. & Intersection number I \\
\hline \hline$a^{\prime} a$ & $A_{a}$ & $\frac{1}{2}\left(\pi_{a}^{\prime} \circ \pi_{a}+\pi_{O 6} \circ \pi_{a}\right)$ \\
$a^{\prime} a$ & $S_{a}$ & $\frac{1}{2}\left(\pi_{a}^{\prime} \circ \pi_{a}-\pi_{O 6} \circ \pi_{a}\right)$ \\
$a b$ & $\left(\bar{N}_{a}, N_{b}\right)$ & $\pi_{a} \circ \pi_{b}$ \\
$a^{\prime} b$ & $\left(N_{a}, N_{b}\right)$ & $\pi_{a}^{\prime} \circ \pi_{b}$ \\
\hline
\end{tabular}

- Since the Ramond charges of the space-time filling D-branes cannot 'escape' to infinite, the internal Ramond charges on compact space must cancel (Gauss law). This is the issue of Ramond tadpole cancellation which give some strong restrictions on the allowed D-brane configurations. Specifically, the Ramond tadpole conditions follow from the equations of motion for the gauge field $C_{7}$ :

$$
\frac{1}{\kappa^{2}} d \star d C_{7}=\mu_{6} \sum_{a} N_{a} \delta\left(\pi_{a}\right)+\mu_{6} \sum_{a} N_{a} \delta\left(\pi_{a}^{\prime}\right)+\mu_{6} Q_{6} \delta\left(\pi_{\mathrm{O} 6}\right),
$$

where $\delta\left(\pi_{a}\right)$ denotes the Poincaré dual form of $\pi_{a}, \mu_{p}=2 \pi\left(4 \pi^{2} \alpha^{\prime}\right)^{-(p+1) / 2}$, and $2 \kappa^{2}=\mu_{7}^{-1}$. Upon integrating over $\mathcal{M}_{6}$ one obtains the RR-tadpole cancellation as equation in homology:

$$
\sum_{a} N_{a}\left(\pi_{a}+\pi_{a}^{\prime}\right)-4 \pi_{\mathrm{O} 6}=0 .
$$

In principle it involves as many linear relations as there are independent generators in $H_{3}\left(\mathcal{M}_{6}, R\right)$. But, of course, the action of $\bar{\sigma}$ on $\mathcal{M}_{6}$ also induces an action $[\bar{\sigma}]$ on the homology and cohomology. In particular, $[\bar{\sigma}]$ swaps $H^{2,1}$ and $H^{1,2}$, and the number of conditions is halved.

- Next, there is the requirement of cancellation of the internal D-brane tensions, i.e the forces between the D-branes must be balanced. In terms of string amplitudes, it means that all NS tadpoles must vanish, namely all NS tadpoles of the closed string moduli fields and also of the dilaton field. Absence of these tadpoles means that the potential of those fields is minimized. The disc level tension can be determined by integrating the DiracBorn-Infeld effective action. It is proportional to the volume of the D-branes and the O-plane, so that the disc level scalar potential reads

$$
\begin{aligned}
\mathcal{V} & =T_{6} \frac{e^{-\phi_{4}}}{\sqrt{\operatorname{Vol}\left(\mathcal{M}_{6}\right)}}\left(\sum_{a} N_{a}\left(\operatorname{Vol}\left(\operatorname{D} 6_{a}\right)+\operatorname{Vol}\left(\mathrm{D} 6_{a}^{\prime}\right)\right)-4 \operatorname{Vol}(\mathrm{O} 6)\right) \\
& =T_{6} e^{-\phi_{4}}\left(\sum_{a} N_{a}\left|\int_{\pi_{a}} \widehat{\Omega}_{3}\right|+\sum_{a} N_{a}\left|\int_{\pi_{a}^{\prime}} \widehat{\Omega}_{3}\right|-4\left|\int_{\pi_{\mathrm{O} 6}} \widehat{\Omega}_{3}\right|\right) .
\end{aligned}
$$

The potential is easily seen to be positive semidefinite and its minimization imposes conditions on some of the moduli, freezing them to fixed values. 
Whenever the potential is non-vanishing, supersymmetry is broken and a classical vacuum energy generated by the net brane tension. It is easily demonstrated that the vanishing of $\mathcal{V}$ requires all the cycles wrapped by the D6-branes to be calibrated with respect to the same 3-form as are the O6-planes.

- One can show that the cancellation of the RR tadpoles implies absence of the non-Abelian anomalies in the effective 4D field theory. However there can be still anomalous $U(1)$ gauge symmetries in the effective $4 \mathrm{D}$ field theory. These anomalies will be canceled by a Green-Schwarz mechanism involving Ramond (pseudo)scalar field. As a result of these interactions the corresponding $U(1)$ gauge boson will become massive. Considering the relevant triangle diagrams the condition for an anomaly free $U(1)_{a}$ is:

$$
N_{a}\left(\pi_{a}-\pi_{a}^{\prime}\right) \circ \pi_{b}=0 .
$$

Note that even an anomaly free $U(1)$ can become massive. The massive $U(1)$ always remains as a global symmetry. For SM engineering, we always have to require that the linear combination of $U(1)$ 's that corresponds to $U(1)_{Y}$ is anomaly free and massless.

- Besides the local triangle anomalies, field theoretical models can be plagued by global $S U(2)$ gauge anomalies. In orientifold models this requirement can be deduced from a K-theory analysis. In the case of our models, this condition requires an even amount of chiral matter from $S p(2)$ probe branes. In this case we obtain the following condition for a model with $k$ stack of branes:

$$
\sum_{a=1}^{k} N_{a} \pi_{a} \circ \pi_{p} \equiv 0 \quad \bmod \quad 2
$$

This equation should hold for any probe brane $p$ invariant under the orientifold map.

To be specific, we now restrict ourselves on orbifold compactifications, i.e. $\mathcal{M}_{6}$ is a toroidal $Z_{N}$ resp. $Z_{N} \times Z_{M}$ orientifold. First, we consider the case $\mathcal{M}_{6}=$ $T^{6} / Z_{2} \times Z_{2}=\prod_{I=1}^{3} T_{I}^{2} / Z_{2} \times Z_{2}$. The D6-branes are wrapping special Langrangian 3 -cycles, which are products of 1 -cycles in each of the three subtori $T_{I}^{2}$. Hence they are characterized by three pairs of integer-valued wrapping numbers $X^{I}, Y^{I}$ $(I=0, \ldots, 3)$. The supersymmetry conditions, being equivalent to the vanishing of the D-term scalar potential $\mathcal{V}$ (see eq.(11)), now have the form:

$$
\sum_{I=0}^{3} \frac{Y^{I}}{U_{I}}=0, \quad \sum_{I=0}^{3} X^{I} U_{I}>0 .
$$

The $U_{I}$ are the three complex structure moduli of the three two-tori $T_{I}^{2}$. The Ramond tadpole cancellation conditions eq.(10) for $k$ stacks of $N_{a}$ D6-branes are now 
given by

$$
\sum_{a=1}^{k} N_{a} \vec{X}_{a}=\vec{L}
$$

where the $L^{I}$ parametrize the orientifold charge. In addition there are some more constraints from K-theory. Chiral matter in bifundamental representations originate from open strings located at the intersection of two stacks of D6-branes with a multiplicity (generation) number given by the intersection number

$$
I_{a b}=\sum_{I=0}^{3}\left(X_{a}^{I} Y_{b}^{I}-X_{b}^{I} Y_{a}^{I}\right) .
$$

Now we come to the statistical properties of this particular class of orientifold compactifications. Specifically, we first want to count all different, consistent Dbrane embeddings into the given $T^{6} / Z_{2} \times Z_{2}$ background geometry. I.e. we want to count all possible solutions of the D-brane equations (14) and (15). These set of equations are diophantic equations in the integer wrapping numbers $X^{I}, Y^{I}$, and they contain as continuous parameters the complex structure moduli $U_{I}$. First we want to know, if for any given tadpole charge $\vec{L}$ there is a finite number of solutions of these equations. Actually, based on a saddle point approximation, the total number of D-brane embeddings can be estimated as follows: ${ }^{66}$

$$
N_{\text {D-branes }}(L) \simeq e^{2 \sqrt{L \log L}} .
$$

For typical orientifold charges like $L=64$, one obtains as estimate that $N_{\mathrm{D} \text {-branes }} \simeq$ $2 \times 10^{9}$.

Next, we explicitly count all possible solutions of the D-brane equations (14) and (15) by running a computer program; this leads to a total of $1.66 \cdot 10^{8}$ supersymmetric D-brane models on the $Z_{2} \times Z_{2}$ orientifold. ${ }^{67-69}$ However this computer count was limited by the available CPU time of about $4 \times 10^{5}$ hours, and hence it could be done only for restricted, not too large values of the complex structure parameters $U_{I}$. However, $i^{70}$ an analytic proof was found that the number of solutions for eqs.(14) and (15) is indeed finite.

With this large sample of models we can ask the question which fraction of models satisfy several phenomenological constraints that gradually approach the spectrum of the supersymmetric MSSM. This is summarized in the following table: 


\begin{tabular}{|l|r|}
\hline Restriction & Factor \\
\hline gauge factor $U(3)$ & 0.0816 \\
gauge factor $U(2) / S p(2)$ & 0.992 \\
No symmetric representations & 0.839 \\
Massless $U(1)_{Y}$ & 0.423 \\
Three generations of quarks $\left(I_{a b}^{\text {quarks }}=3\right)$ & $2.92 \times 10^{-5}$ \\
Three generations of leptons $\left(I_{a b}^{\text {leptons }}=3\right)$ & $1.62 \times 10^{-3}$ \\
\hline Total & $1.3 \times 10^{-9}$ \\
\hline
\end{tabular}

The total probability of $1.3 \times 10^{-9}$ is simply obtained multiplying each probability factors in the first six rows, since one can show that there is little correlation between these individual probabilties. We see that statistically only one in a billion models give rise to an MSSM like D-brane vacuum. Multiplying this result with the initial number of models, the chance to find the MSSM is less then one. One can now compare this statistical result with the explicitly constructed intersecting D6-brane models with MSSM like spectra ( see $^{64}$ for references on various intersecting D-brane models). In fact, in ${ }^{71}$ a $Z_{2} \times Z_{2}$ orientifold model with MSSM like spectrum was found that should be contained in the statistical search discussed above. However unfortunately this model is outside the range of complex structure moduli covered by our computer scan. Also note that all $Z_{2} \times Z_{2}$ MSSM like models found so far contain also exotic particles, not present in the MSSM. These exotic particles were allowed in our statistical search.

The statistical scan was extended in ${ }^{72}$ to the case of the $Z_{6}$ orbifold geometry background geometry. For this class of orbifold backgrounds explicit MSSM like models were constructed in. ${ }^{73}$ Compared to the $Z_{2} \times Z_{2}$ orientifold, the $Z_{6}$ case is more complex, because it also contains exceptional, twisted (blowing-up) 3-cycles, besides the untwisted bulk 3-cycles. D6-branes wrapped around the exceptional 3cycles correspond to fractional branes. First, it was possible to show that even in the presence of the exceptional cycles the number of the D-brane solutions of the tadpole plus supersymmetry conditions is finite. Then, by extended computer scan it was found that there exist $3.4 \times 10^{28}$ solutions in total, of which $5.7 \times 10^{6}$ contain the gauge group and the chiral matter content of the MSSM. We therefore obtained a probability of $1.7 \times 10^{-22}$ to find MSSM like vacua, a number considerably lower than the value $10^{-9}$ for the case of the $Z_{2} \times Z_{2}$ orientifolds.

Finally, similar results can be obtained for orbifold models with $S U(5)$ GUT gauge group. ${ }^{74}$

Complementary to the orbifold models, there are the intersecting brane constructions based on rational conformal field theories, in particular Gepner models ${ }^{75,76}$ $\mathrm{In}^{77,78}$ an extended investigation of the statistics of Gepner models orientifolds was presented. Here the likelihood to find MSSM like solutions based on the 4-stack quiver shown before within all brane solutions is about $10^{-12}$. Note however that in 
this Gepner model scan exotic representations were excluded. Recently, the search for D-instanton generated righthanded Majorana neutrino masses was included into the Gepner model statistics. ${ }^{79}$

Finally let us mention that other statistical aspects of the D-brane landscape were discussed in. ${ }^{80}$

\section{Moduli stabilization}

In this section we discuss a few aspects about the moduli stabilization process due to background fluxes (see ref. ${ }^{81,82}$ for reviews on flux compactifications) and nonperturbative effects. The number of flux vacua on a given CY background space is very huge: ${ }^{7,8,83} \mathcal{N}_{\text {vac }} \sim 10^{500}$. Again one can try to make some interesting statistical predictions within the flux landscape, like the question what is the likelihood for obtaining a tiny cosmological constant, or if supersymmetry is broken at high or low energy scales. ${ }^{84-87}$ Of course, in order to make more concrete predictions in the string landscape, the flux vacua statistics must be eventually combined with the D-brane statistics, described before.

To be specific we discuss type IIB compactifications, which include D3/D7branes and the associated O3/O7-planes (class (ii) brane models). First consider Ramond and NS 3-form fluxes through 3-cycles of a Calabi-Yau space $\mathcal{M}_{6}$. They give rise to the following effective flux superpotential in four dimensions: ${ }^{88-90}$

$$
W_{\text {flux }}(\tau, U) \sim \int_{\mathcal{M}_{6}}\left(H_{3}^{R}+\tau H_{3}^{N S}\right) \wedge \Omega .
$$

$W_{\text {flux }}$ depends on the dilaton $\tau$ and also on the complex structure moduli $U$. However, since $W_{\text {flux }}$ does not depend on the Kähler moduli, one needs additional nonperturbative contributions to the superpotential in order to stabilize all moduli, as it was proposed in the $\mathrm{KKLT}^{91}$ scenario. In the following we want to address the question, what are the restrictions to the KKLT scenario in order to be implemented in CY compactifications, and on which concrete CY spaces these constraints can be satisfied, such that all moduli can be indeed fixed.

The non-perturbative part of the KKLT superpotential is provided by Euclidean D3-instantons, ${ }^{92}$ which are wrapped around 4 -cycles (divisors) $D$ inside $\mathcal{M}_{6}$, and/or gaugino condensations in hidden gauge group sectors on the world volumes of D7branes, which are also wrapped around certain divisors $D$. Both give rise to terms in the superpotential of the form

$$
W_{\text {n.p. }} \sim g_{i} \Phi^{n} e^{-a_{i} V_{i}},
$$

where $V_{i}$ is the volume of the divisor $D_{i}$, depending on the Kähler moduli $T$. The fields $\Phi$ are matter fields in bifundamental representations that are located at the intersections of space-time filling D7-branes, which are at the same time also intersected by the D3-instantons, resp. the D3-instantons lie on top of the D7-branes. Their presence is in general required in order to make the non-perturbative superpotential invariant under global $U(1)$ symmetries, which are remnants of local $U(1)$ 
gauge symmetries that became massive by the GS-mechanism. In fact, the $U(1)$ invariance of $W_{\text {n.p }}$ can be verified by knowing the transformation properties of the moduli $V_{i}$ (shift symmetries) and the matter fields $\Phi$ under the $U(1)$ transformations. ${ }^{93-95}$ For a pure D3-instanton, which is not intersected by any D7-brane, there are no matter fields in $W_{\text {n.p. }}$, i.e. $n=0$.

For gaugino condensation in a hidden gauge group, $V_{i}$ is the (holomorphic) gauge coupling constant of $G_{\text {hidden }}$, and $W_{\text {n.p. }}$ corresponds to the field theory Affleck-DineSeiberg/Taylor-Veneziano-Yankielowicz (ADS/TVY) superpotential. ${ }^{96,97}$ Here the number of matter fields in $W_{\text {n.p. }}$ is determined by the number of colors and flavors of $G_{\text {hidden }}$. In the simplest case with $G_{\text {hidden }}=S U\left(N_{C}\right)$ and $N_{F}=N_{C}-1, W_{\text {n.p. }}$ is induced by a single D3-instanton that is wrapping the same 4-cycle as the $N_{c}$ gauge D7-branes.

Note that the pre-factor $g_{i}$ is in general not a constant, but rather depends on the complex structure moduli $U .{ }^{98}$ In fact, in case of gauge instantons resp. gaugino condensation $g_{i}$ is related to the holomorphic part of the one-loop gauge threshold corrections $\Delta_{1-\text { loop }}(U)$ in D-brane models: ${ }^{99,100}$

$$
g_{i} \simeq \exp (\Delta(U)) .
$$

Explicit calculations in type IIA intersecting D6-brane models give for $\mathcal{N}=2$ supersymmetric brane sectors that (here in type IIB notation)

$$
\Delta(U) \simeq \log \eta(U),
$$

whereas in $\mathcal{N}=1$ brane sectors the holomorphic threshold corrections is just a constant. However note that the full, 1-loop threshold contain additional nonholomorphic, moduli dependent contributions. They are due to $\sigma$-model anomalies, and are related to the tree level Kähler potential of the matter fields. ${ }^{101,102}$

The generation of a non-perturbative superpotential crucially depends on the D-brane zero modes of the wrapping divisors, i.e. on the topology of the divisors together with their interplay with the O-planes and also with the background fluxes. ${ }^{103-106}$ In case there are too many zero modes (too much supersymmetry is preserved on the divisor) the non-perturbative superpotential is simply absent. In gauge theory language, additional (bosonic) zero modes correspond to massless adjoint matter fields, which make gaugino condensation impossible,

It is worth mentioning that recently it became possible to compute the Dinstanton generated superpotential by open string CFT methods, ${ }^{94,107-115}$ namely by computing open string amplitudes between zero mode fields, which correspond to open strings with ends on the internal Euclidean D-branes, and physical matter fields, corresponding to open strings with both ends on space-time filling Dbranes. These CFT computations can be applied for deriving the non-perturbative ADS/TVY superpotential from D-instantons (for the case $N_{F}=N_{C}-1$ in $S U\left(N_{C}\right)$ gauge theories with $N_{F}$ vector-like fundamental matter fields, and also for $S p\left(N_{C}\right)$ with $N_{F}=N_{C}$ fundamental matter fields, and for $S O\left(N_{C}\right)$ with $N_{F}=N_{C}-3$ ), and hence are relevant for moduli stabilization. In addition, the D-instantons can 
also generate new matter couplings, like righthanded Majorana neutrino masses, Yukawa couplings, FI-terms or non-perturbative contributions to the gauge kinetic functions, which are classically forbidden by the massive $U(1)$ symmetries mentioned above.

Let us come back to the problem of moduli stabilization. In the low energy effective supergravity theory, the moduli are stabilized to discrete values by solving the $\mathcal{N}=1$ supersymmetry conditions

$$
D_{A} W=0 \quad \text { (vanishing } \mathrm{F}-\text { term). }
$$

Then typically the superpotentials of the form $W_{\text {flux }}+W_{\text {n.p. }}$ lead to stable supersymmetric $A d S_{4}$ minima. Additional restrictions on the form of the superpotential arise $^{116,117}$ requiring that the mass matrix of all the fields $(S, T, U)$ is already positive definite in the AdS vacuum (absence of tachyons), as it is necessary, if one wants to uplift the AdS vacua to a dS vacuum by a shift in the potential. These conditions cannot be satisfied in orientifold models without any complex structure moduli, i.e. for Calabi-Yau spaces with Hodge number $h^{2,1}=0$. Alternatively one can also look for supersymmetric 4D Minkowski minima which solve eq.(22). ${ }^{118,119}$ They may exist if $W_{\text {n.p. }}$ is of the racetrack form. In this case the requirement that all flat directions are lifted in the Minkowski vacuum leads to similar constraints as the absence of tachyon condition in the AdS case.

In more concrete terms, the moduli stabilization procedure to $A d S_{4}$ vacua was studied in ${ }^{120}$ for the $T^{6} / Z_{2} \times Z_{2}$ orientifold, with the result that all moduli indeed can be fixed. Moreover in ${ }^{117,121-125}$ all other $Z_{N}$ and $Z_{N} \times Z_{M}$ orientifolds were studied in great detail, where it turns out that in order to have divisors, which contribute to the non-perturbative superpotential, one has to consider the blownup orbifold geometries. Then the divisors originating from the blowing-ups give rise to D3-instantons and/or gaugino condensates, being rigid and hence satisfying the necessary topological conditions. As a result of this investigation of all possible orbifold models, it turns out that the $Z_{2} \times Z_{2}, Z_{2} \times Z_{4}, Z_{4}, Z_{6-I I}$ orientifolds are good candidates where all moduli can be completely stabilized to a supersymmetric ADS vacuum with positive definite scalar mass matrix.

The up-lift to a vacuum with positive cosmological constant (dS-vacuum) and the breaking of space-time supersymmetry can be done in several ways. First, the original KKLT scenario ${ }^{91}$ proposes to introduce anti-D3-branes, which explicitly break supersymmerty. A second proposal deals with D7-branes that contain additional supersymmetry breaking F-flux on their world volumes. In field theory language this corresponds to up-lifting and supersymmetry breaking by a D-term scalar potential. ${ }^{93,95,126-129}$ Since in a non-supersymmetric supergravity groundstate, Fand D-terms are proportional to each other, the non-vanishing D-term potential implies also the existence of a non-vanishing F-term, i.e. the original F-term SUSY condition eq. (22) of the ADS-vacuum has to be relaxed. Another interesting issue

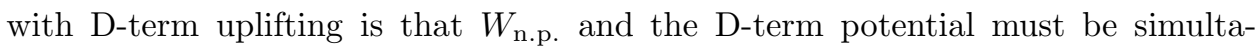
neously invariant under the massive $U(1)$ gauge symmetries, which is indeed the 
case after including the matter fields in $W_{\text {n.p. }}$. Alternatively, up-lifting by F-terms was discussed in the literature, ${ }^{130,131}$ where again matter fields play an important rule. Moreover, the existence of recently discovered metastable, non-supersymmetric vacua in supersymmetric gauge theories ${ }^{132}$ and in their string realizations ${ }^{133,134}$ most likely will become important for the up-lifting procedure. However many details of the up-lifting procedure still have to worked out in concrete models.

After breaking space-time supersymmetry the soft SUSY breaking parameters for the matter fields on the D-branes can be computed. ${ }^{135-145}$ It will become important to compare the pattern of soft parameters obtained in D-brane models with the experimental data, which we will hopefully obtain from the LHC in the next years. E.g. the analysis in ${ }^{139}$ indicates that the squark masses are heavier that the gaugino masses on the D7-branes.

\section{Black hole/flux correspondence and probability wave function}

In this section we like to discuss a method to assign a probability measure to flux compactifications via an associated black hole entropy functional $\mathcal{S}$. As mentioned in the introduction, it is possible to compute the thermodynamic Bekenstein-Hawking entropy of charged, supersymmetric black holes in string theory by counting the associated microscopic string degrees of freedom (see ref. ${ }^{1}$ for a black hole review). To be specific, we like consider charged, supersymmetric black holes in $\mathcal{N}=2$ supergravity, which arise by compactifying type II A/B superstrings on a Calabi-Yau manifold. The four-dimensional black holes carry magnetic/electric $U(1)$ charges, denoted by $p^{I}$ and $q_{I}$, with respect to the $4 \mathrm{D}$ Abelian field strength $F_{I}$ and $G^{I}$ $(I=0, \ldots N)$ :

$$
q_{I}=\oint_{S^{2}} F_{I}, \quad p^{I}=\oint_{S^{2}} G^{I}
$$

where the 2-sphere $S^{2}$ is embedded into the four-dimensional space time. In $\mathcal{N}=2$ supergravity, these $N+1$ fields strengths fields are components of $N$ vector multiplets plus the graviphoton field strength. In type IIB compactifications, the $\mathcal{N}=2$ vector multiplets correspond to the complex structure deformations, i.e. $N=h^{2,1}$, whereas in type IIA they are related to the Kähler deformations, and therefore $N=h^{1,1}$. In the following we concentrate on the type IIB case. Here, the fourdimensional field strength fields arise by dimensionally reducing the type IIB Ramond 5-form field strength $F_{5}$ on internal internal three cycles $\Sigma_{I} \subset \mathcal{M}_{6}$ and $\tilde{\Sigma}^{I} \subset \mathcal{M}_{6}$ :

$$
F_{I}=\oint_{\Sigma_{I}} F_{5}, \quad G^{I}=\oint_{\Sigma^{I}} F_{5}
$$

The charged black holes have the interpretation of D3-brane, which are the sources of the 5-form field strengths $F_{5}$, and which are wrapped around these 3-cycles.

In four dimensional supergravity, the supersymmetric, charged blackholes are interpolating solutions that interpolate between flat space at radial infinity and the 
$A d S_{2} \times S^{2}$ geometry at the horizon of the black hole. At the horizon, full $\mathcal{N}=$ 2 space-time supersymmetry is restored. Furthermore, according to the attractor mechanism, the values of the scalar fields $\phi_{A}$ (complex structure moduli) at the horizon are determined by the supersymmetry condition (stabilization condition)

$$
D_{A} Z(\phi)=0 \quad \Longrightarrow \quad \phi_{A, \text { hor. }}
$$

where $Z$ is the central charge of the $\mathcal{N}=2$ supersymmetry algebra. Solving this condition, it follows that the $\phi_{A \text {,hor. }}$ are entirely given in terms of the magnetic/electric charges $\left(p^{I}, q_{I}\right)$ :

$$
\phi_{A, \text { hor. }}=\phi_{A}\left(p^{I}, q_{I}\right), \quad A=1, \ldots, N .
$$

Finally, the Bekenstein-Hawking entropy of the charged black holes is also a function of the charges $\left(p^{I}, q_{I}\right)$, as it is given in terms of the value of the central charge $Z$ at the horizon:

$$
\mathcal{S}_{b h}(\phi(p, q))=|Z(\phi(p, q))|^{2}=\frac{A_{\text {hor }}(\phi(p, q))}{4} .
$$

Using the attractor relation between charges $\left(p^{I}, q_{I}\right)$ and scalar moduli fields, eq.(26), the black hole entropy can be also regarded as a functional over the complex structure moduli space:

$$
\mathcal{S}_{b h}=\mathcal{S}(\phi) .
$$

However it is important to note that the attractor relation eq.(26) is not unique, i.e. one-to-one, since there is one more pair of charges $\left(p^{I}, q_{I}\right)$ compared the number of scalar fields $\phi_{A}$. Hence to compute the entropy functional $\mathcal{S}(\phi)$ from the attractor equations, one electric and one magnetic charge has to be fixed by hand.

The black hole attractor equations are quite similar to the supersymmetry conditions eq.(22) in the context of flux compactifications. ${ }^{146,147}$ Therefore one might conjecture a close connection between the string flux landscape and the entropy of charged black holes. In fact, instead of 3-form flux vacua in four dimensions, Ooguri, Verlinde, Vafa $(\mathrm{OVV})^{148,149}$ considered Ramond 5-form flux compactifications on $S^{2} \times \mathcal{M}_{6}$ to two dimensions. The corresponding 2-dimensional, supersymmetric vacua, which follow from a supersymmetry condition

$$
D W=0, \quad W \sim \int_{S^{2} \times \mathcal{M}_{6}}\left(F_{5} \wedge \Omega\right),
$$

have $A d S_{2}$ geometry. This supersymmetry condition is formally the same as the stabilization condition $D Z=0$ of the $4 \mathrm{D}$ charged black holes discussed above. In view of this connection, it was suggested by OVV that the entropy functional of the $4 \mathrm{D}$ black holes determined the wave function of the $2 \mathrm{D}$ flux vacua, namely to interpret

$$
\psi(\phi)=e^{\mathcal{S}(\phi)}
$$

as a probability distribution resp. wave function for $2 \mathrm{D}$ flux compactifications. So the probability functions is peaked at points of maximal entropy in the moduli 
space. In a stringy picture, $\psi$ essentially counts the microscopic string degrees of freedom, which are associated to each flux vacuum.

The correspondence between flux vacua and charged black holes can be summarized by the following table:

\begin{tabular}{c|c} 
Black holes & Flux compactifications \\
\hline D3-branes wrapped around $\Sigma_{3}$ & $F_{5}$ through $S^{2} \times \Sigma_{3}$ \\
Black hole charges $\left(q_{I}, p^{I}\right)$ & 5 -form fluxes $\left(q_{I}, p^{I}\right)$ \\
Central charge $Z(\phi)$ & Superpotential $W(\phi)$ \\
Stabilization cond. $D_{A} Z=0$ & Supersymmetry cond. $D_{A} W=0$ \\
Entropy $\mathcal{S}$ & Cosmological constant $-\Lambda$ \\
Near horizon geometry $A d S_{2} \times S^{2}$ & Vacuum space $A d S_{2} \times S^{2}$
\end{tabular}

As can be seen from this table, the black hole entropy corresponds to the negative value of the $A d S_{2}$ cosmological constant in the flux landscape. Hence one obtains for the wave function $\psi$ the following relation:

$$
\psi(\phi)=e^{-\Lambda(\phi)}
$$

This form of the wave function is very similar to the Hartle-Hawking wave function, which describes certain tunneling amplitudes between different vacua. It implies that $A d S_{2}$ vacua with minimal cosmological constant possess the largest probability.

The principle of entropy maximization was further studied in. ${ }^{149-151}$ As a simple toy example the entropy functional near the special point in Calabi-Yau compactifications, where new states become massless (conifold point), was studied. In particular, the following question was addressed, namely is the entropy functional maximal resp. minimal in case $\mathcal{N}=2$ vector/hyper multiplets become massless at this special point? For massless hypermultiplets, the effective gauge theory at the conifold is infrared free (negative $\beta$-function coefficient, i.e. $\beta<0$, and for massless vector multiplets the effective gauge theory is asymptotically free, i.e. $\beta>0$.

The answer to the question above is the following. Using the known expression for the $\mathcal{N}=2$ prepotential near the conifold point, the entropy functional takes the following form

$$
\mathcal{S}(\phi)=\text { const. }-\frac{\beta}{2 \pi}(\operatorname{Re} \phi)^{2}-\frac{2 \beta}{\pi}|\phi|^{2} \log |\phi| .
$$

Here $\phi$ is the complex structure modulus, which describes the moduli space in the neighborhood of the conifold point. From this formula it follows that the entropy is maximal at $\phi=0$ for $\beta<0$ and minimal for $\beta>0^{151}$. Hence, according to this discussion the effective, infrared free gauge theory is more probable than the asymptotically free gauge theory. This conclusion also persists, if one includes higher curvature corrections in the entropy functional, or if one uses the non-perturbative prepotential from topological string theory. ${ }^{152}$

$\mathrm{In}^{153}$ the entropy functional of non-supersymmetric, charged black holes around the conifold point was studied. In turns out that in this case the conifold point is 
not anymore an attractor point. However one can show that there exist an attractor point in the close neighborhood of the conifold point.

Finally let us end with some comments on a probability functional of 4dimensional $A d S_{4}$ flux vacua. Supersymmetric $A d S_{4}$ vacua with all moduli fixed can be constructed in type IIA flux compactifications with 0,2,4,6-form Ramond fluxes plus possibly geometrical fluxes, ${ }^{154-159}$ and also in type IIB on some non-geometrical background spaces with 3 -form fluxes. ${ }^{160,161}$ In the next step one replaces the various fluxes by their corresponding smeared brane sources. ${ }^{162}$ These branes are wrapping internal cycles, and they are domain walls in the four-dimensional space time, like D4-branes wrapped around internal 2-cycles, D8-branes wrapped around the CY-space or NS5-branes wrapped around internal 3-cycles. As one can show, ${ }^{162}$ these brane configurations are supersymmetric, create the same tadpoles as the fluxes, and in $4 \mathrm{D}$ their interpolate between the near horizon geometry $A d S_{4}$ and flat Minkowski space-time. So having constructed these supersymmetric brane configurations that correspond to $A d S_{4}$ flux vacua, one might hope to determine thei entropies of these 4D domain walls and to get in this way a probability functional in the four-dimensional flux landscape.

\section{Final comments}

In this talk we addressed the question how to relate the string theory landscape with low energy physics, in particular with the MSSM. Phenomenologically interesting heterotic constructions and D-brane models were discussed. In particular starting from a large sample of consistent D-brane embeddings into a given 6-dimensional internal space, we investigated the statistical likelihood of obtaining models which come to the MSSM. In additions, some mechanisms for moduli stabilization were investigated, where we also addressed the question whether these mechanisms can be successfully be realized in concrete orientifold compactifications. One caveat in this discussion is that the moduli stabilization occurs within the effective supergravity approach which is limited to large volumes of the internal spaces. It would be interesting to learn more about the string landscape taking into account higher order $\alpha^{\prime}$ corrections, or investigating the problem of the string ground state in a background independent formulation. Finally, some ideas about probability functions in the string moduli space in relation with black hole entropies were presented. This apparently works for two-dimensional $A d S_{2}$ flux vacua, which are related to 4D charged black hole solutions. Whether a similar constructions also works for 4-dimensional $A d S_{4}$ flux vacua, which can be related to $4 \mathrm{D}$ domain wall solutions, still has to be seen in the future. For the string landscape discussion it is also important to learn what kind of field theory are inside the string landscape and can be consistently coupled to gravity, and which field theories lie outside the string landscape, the socalled swampland. ${ }^{163}$

During the next years, we hope to get a lot of new informations for string model building from new experiments, most notable the LHC and new astrophysical ob- 
servations. It will be very important to concretely work out the chain between the experimental data and the string theory parameters, which are to large extent given in terms of geometrical and topological parameters of the internal compact spaces. At the moment string theory still does not make too many very explicit predictions about soft supersymmetry breaking masses etc. However there are important qualitative predictions from string theory. In particular we are interested in the answer to the following questions:

- Where is the intrinsic string scale $M_{\text {string? }}$ ?

- What is the size of the extra dimenensions?

- What is the scale of supersymmetry breaking?

To answer these questions is indeed a great challenge for the next years; to make progress in our understanding about string theory or more generally about physics at very short distances needs big combined efforts of mathematicians, string theorists, particle phenomenologists, astrophysicists and last but not least of experimentalists.

\section{Acknowledgments}

It is a great pleasure to thank my collaborators R. Blumenhagen, G. Lopes Cardoso, G. Curio, F. Gmeiner, V. Grass, M. Haack, G. Honecker, B. Körs, D. Krefl, J. Perz, S. Reffert, E. Scheidegger, W. Schulgin, S. Stieberger, A. Van Proeyen, D. Tsimpis, T. Weigand and M. Zagermann for fruitful and pleasant collaboration on the material presented in this paper. This work is supported in part by the European Community's Human Potential Program under contract MRTN-CT-2004005104 'Constituents, fundamental forces and symmetries of the universe' and also by 'The Cluster of Excellence for Fundamental Physics - Origin and Structure of the Universe' in München 


\section{References}

1. T. Mohaupt, "Supersymmetric black holes in string theory," arXiv:hep-th/0703035.

2. J. M. Maldacena, "The large N limit of superconformal field theories and supergravity," Adv. Theor. Math. Phys. 2, 231 (1998) [Int. J. Theor. Phys. 38, 1113 (1999)] arXiv:hep-th/9711200.

3. E. Witten, "Anti-de Sitter space and holography," Adv. Theor. Math. Phys. 2, 253 (1998) arXiv:hep-th/9802150.

4. S. S. Gubser, I. R. Klebanov and A. M. Polyakov, "Gauge theory correlators from non-critical string theory," Phys. Lett. B 428, 105 (1998) arXiv:hep-th/9802109.

5. N. Arkani-Hamed, S. Dimopoulos and G. R. Dvali, Phys. Lett. B 429, 263 (1998), hep-ph/9803315.

6. W. Lerche, D. Lüst and A. N. Schellekens, "Chiral Four-Dimensional Heterotic Strings From Selfdual Lattices," Nucl. Phys. B 287 (1987) 477.

7. R. Bousso and J. Polchinski, "Quantization of four-form fluxes and dynamical neutralization of the cosmological constant," JHEP 0006 (2000) 006 arXiv:hep-th/0004134.

8. M. R. Douglas, "The statistics of string / M theory vacua," JHEP 0305, 046 (2003) arXiv:hep-th/0303194.

9. S. Weinberg, "Anthropic Bound on the Cosmological Constant," Phys. Rev. Lett. 59, 2607 (1987)

10. L. Susskind, "The anthropic landscape of string theory," arXiv:hep-th/0302219

11. A. N. Schellekens, "The landscape 'avant la lettre'," arXiv:physics/0604134

12. A. Linde, "Inflationary Cosmology," arXiv:0705.0164 [hep-th].

13. J. B. Hartle and S. W. Hawking, "Wave Function Of The Universe," Phys. Rev. D 28, 2960 (1983).

14. B. de Carlos, J. A. Casas, F. Quevedo and E. Roulet, "Model independent properties and cosmological implications of the dilaton and moduli sectors of 4-d strings," Phys. Lett. B 318, 447 (1993) arXiv:hep-ph/9308325.

15. R. Friedman, J. W. Morgan and E. Witten, "Vector Bundles Over Elliptic Fibrations," arXiv:alg-geom/9709029

16. B. A. Ovrut, T. Pantev and R. Reinbacher, "Torus-fibered Calabi-Yau threefolds with non-trivial fundamental group," JHEP 0305, 040 (2003) arXiv:hep-th/0212221.

17. V. Braun, B. A. Ovrut, T. Pantev and R. Reinbacher, "Elliptic Calabi-Yau threefolds with Z(3) x Z(3) Wilson lines," JHEP 0412, 062 (2004) arXiv:hep-th/0410055.

18. V. Braun, Y. H. He, B. A. Ovrut and T. Pantev, "A heterotic standard model," Phys. Lett. B 618, 252 (2005) arXiv:hep-th/0501070.

19. B. A. Ovrut, "A Heterotic Standard Model," AIP Conf. Proc. 805, 236 (2006).

20. B. Andreas, "On vector bundles and chiral matter in $\mathrm{N}=1$ heterotic JHEP 9901 , 011 (1999) arXiv:hep-th/9802202.

21. G. Curio, "Chiral matter and transitions in heterotic string models," Phys. Lett. B 435, 39 (1998) arXiv:hep-th/9803224.

22. B. Andreas, G. Curio and A. Klemm, "Towards the standard model spectrum from elliptic Calabi-Yau," Int. J. Mod. Phys. A 19, 1987 (2004) arXiv:hep-th/9903052.

23. G. Curio, "Standard model bundles of the heterotic string," Int. J. Mod. Phys. A 21, 1261 (2006) arXiv:hep-th/0412182.

24. B. Andreas and G. Curio, "Standard models from heterotic string theory," arXiv:hep-th/0602247

25. B. Andreas and G. Curio, "Deformations of Bundles and the Standard Model," arXiv:0706.1158 [hep-th].

26. R. Blumenhagen, G. Honecker and T. Weigand, "Supersymmetric (non-)abelian bundles in the type I and SO(32) heterotic string," JHEP 0508, 009 (2005) 
arXiv:hep-th/0507041.

27. R. Blumenhagen, S. Moster and T. Weigand, "Heterotic GUT and standard model vacua from simply connected Calabi-Yau manifolds," Nucl. Phys. B 751, 186 (2006) arXiv:hep-th/0603015.

28. R. Blumenhagen, S. Moster, R. Reinbacher and T. Weigand, "Massless spectra of three generation $\mathrm{U}(\mathrm{N})$ heterotic string vacua," arXiv:hep-th/0612039

29. T. Weigand, "Compactifications of the heterotic string with unitary bundles," Fortsch. Phys. 54, 963 (2006).

30. L. J. Dixon, V. Kaplunovsky and J. Louis, "Moduli dependence of string loop corrections to gauge coupling constants," Nucl. Phys. B 355, 649 (1991).

31. L. E. Ibanez, D. Lüst and G. G. Ross, "Gauge Coupling Running In Minimal SU(3) X SU(2) X U(1) Superstring Unification," Phys. Lett. B 272, 251 (1991) arXiv:hep-th/9109053.

32. L. E. Ibanez and D. Lüst, Nucl. Phys. B 382, 305 (1992) arXiv:hep-th/9202046.

33. H. P. Nilles and S. Stieberger, "How to Reach the Correct $\sin 2 \theta_{W}$ and $\alpha_{S}$ in String Theory," Phys. Lett. B 367, 126 (1996) arXiv:hep-th/9510009.

34. E. Witten, "Strong Coupling Expansion Of Calabi-Yau Compactification," Nucl. Phys. B 471, 135 (1996) arXiv:hep-th/9602070.

35. H. P. Nilles and S. Stieberger, "String unification, universal one-loop corrections and strongly coupled heterotic string theory," Nucl. Phys. B 499, 3 (1997) arXiv:hep-th/9702110.

36. K. R. Dienes, "String Theory and the Path to Unification: A Review of Recent Developments," Phys. Rept. 287, 447 (1997) arXiv:hep-th/9602045.

37. A. Strominger, "Superstrings with Torsion," Nucl. Phys. B 274, 253 (1986).

38. G. Lopes Cardoso, G. Curio, G. Dall'Agata, D. Lüst, P. Manousselis and G. Zoupanos, "Non-Kähler string backgrounds and their five torsion classes," Nucl. Phys. B 652, 5 (2003) arXiv:hep-th/0211118.

39. K. Becker, M. Becker, K. Dasgupta and P. S. Green, "Compactifications of heterotic theory on non-Kaehler complex manifolds. I," JHEP 0304, 007 (2003) arXiv:hep-th/0301161.

40. K. Becker, M. Becker, P. S. Green, K. Dasgupta and E. Sharpe, "Compactifications of heterotic strings on non-Kaehler complex manifolds. II," Nucl. Phys. B 678, 19 (2004) arXiv:hep-th/0310058.

41. G. Curio, A. Krause and D. Lüst, "Moduli stabilization in the heterotic / IIB discretuum," Fortsch. Phys. 54, 225 (2006) arXiv:hep-th/0502168.

42. V. Braun and B. A. Ovrut, "Stabilizing moduli with a positive cosmological constant in heterotic M-theory," JHEP 0607, 035 (2006) arXiv:hep-th/0603088.

43. S. Raby, "Three family models from the heterotic string," AIP Conf. Proc. 805, 231 (2006) arXiv:hep-th/0510014.

44. W. Buchmüller, K. Hamaguchi, O. Lebedev and M. Ratz, "Supersymmetric standard model from the heterotic string," Phys. Rev. Lett. 96, 121602 (2006) arXiv:hep-ph/0511035.

45. W. Buchmüller, K. Hamaguchi, O. Lebedev and M. Ratz, "Supersymmetric standard model from the heterotic string. II," arXiv:hep-th/0606187

46. A. E. Faraggi, C. Kounnas, S. E. M. Nooij and J. Rizos, "Towards the classification of Z(2) x Z(2) fermionic models," arXiv:hep-th/0311058

47. A. E. Faraggi, C. Kounnas, S. E. M. Nooij and J. Rizos, "Classification of the chiral $\mathrm{Z}(2) \times \mathrm{Z}(2)$ fermionic models in the heterotic superstring," Nucl. Phys. B 695, 41 (2004) arXiv:hep-th/0403058.

48. A. E. Faraggi, C. Kounnas and J. Rizos, "Chiral family classification of 
fermionic Z(2) x Z(2) heterotic orbifold models," Phys. Lett. B 648, 84 (2007) arXiv:hep-th/0606144.

49. K. R. Dienes, "Statistics on the heterotic landscape: Gauge groups and cosmological constants of four-dimensional heterotic strings," Phys. Rev. D 73, 106010 (2006) arXiv:hep-th/0602286.

50. O. Lebedev, H. P. Nilles, S. Raby, S. Ramos-Sanchez, M. Ratz, P. K. S. Vaudrevange and A. Wingerter, "Low Energy Supersymmetry from the Heterotic Landscape," Phys. Rev. Lett. 98, 181602 (2007) arXiv:hep-th/0611203.

51. K. R. Dienes, M. Lennek, D. Senechal and V. Wasnik, "Supersymmetry versus Gauge Symmetry on the Heterotic Landscape," Phys. Rev. D 75, 126005 (2007) arXiv:0704.1320 [hep-th]].

52. C. Bachas, "A Way to break supersymmetry," arXiv:hep-th/9503030

53. R. Blumenhagen, L. Görlich, B. Körs and D. Lüst, "Noncommutative compactifications of type I strings on tori with magnetic background flux," JHEP 0010, 006 (2000) arXiv:hep-th/0007024.

54. C. Angelantonj, I. Antoniadis, E. Dudas and A. Sagnotti, "Type-I strings on magnetised orbifolds and brane transmutation," Phys. Lett. B 489, 223 (2000) arXiv:hep-th/0007090.

55. G. Aldazabal, S. Franco, L. E. Ibanez, R. Rabadan and A. M. Uranga, "D = 4 chiral string compactifications from intersecting branes," J. Math. Phys. 42, 3103 (2001) arXiv:hep-th/0011073.

56. M. Cvetic, G. Shiu and A. M. Uranga, "Three-family supersymmetric standard like models from intersecting brane worlds," Phys. Rev. Lett. 87, 201801 (2001) arXiv:hep-th/0107143.

57. A. Font, L. E. Ibanez and F. Marchesano, "Coisotropic D8-branes and modelbuilding," JHEP 0609, 080 (2006) arXiv:hep-th/0607219.

58. L. E. Ibanez, F. Marchesano and R. Rabadan, "Getting just the standard model at intersecting branes," JHEP 0111, 002 (2001) arXiv:hep-th/0105155.

59. R. Blumenhagen, B. Körs, D. Lüst and T. Ott, "The standard model from stable intersecting brane world orbifolds," Nucl. Phys. B 616, 3 (2001) arXiv:hep-th/0107138.

60. C. Angelantonj and A. Sagnotti, "Open strings," Phys. Rept. 371, 1 (2002) [Erratumibid. 376, 339 (2003)] arXiv:hep-th/0204089.

61. E. Kiritsis, "D-branes in standard model building, gravity and cosmology," Fortsch. Phys. 52, 200 (2004) [Phys. Rept. 421, 105 (2005 ERRAT,429,121-122.2006)] arXiv:hep-th/0310001.

62. D. Lüst, "Intersecting brane worlds: A path to the standard model?," Class. Quant. Grav. 21, S1399 (2004) arXiv:hep-th/0401156.

63. R. Blumenhagen, M. Cvetic, P. Langacker and G. Shiu, "Toward realistic intersecting D-brane models," Ann. Rev. Nucl. Part. Sci. 55, 71 (2005) arXiv:hep-th/0502005.

64. R. Blumenhagen, B. Kors, D. Lüst and S. Stieberger, "Four-dimensional String Compactifications with D-Branes, Orientifolds and Fluxes," arXiv:hep-th/0610327

65. F. Marchesano, "Progress in D-brane model building," arXiv:hep-th/0702094

66. R. Blumenhagen, F. Gmeiner, G. Honecker, D. Lüst and T. Weigand, "The statistics of supersymmetric D-brane models," Nucl. Phys. B $\mathbf{7 1 3}$ (2005) 83 arXiv:hep-th/0411173.

67. F. Gmeiner, R. Blumenhagen, G. Honecker, D. Lüst and T. Weigand, "One in a billion: MSSM-like D-brane statistics," JHEP 0601 (2006) 004 arXiv:hep-th/0510170.

68. F. Gmeiner, "Standard model statistics of a type II orientifold," arXiv:hep-th/0512190

69. F. Gmeiner, "Gauge sector statistics of intersecting D-brane models," Fortsch. Phys. 
55, 111 (2007) arXiv:hep-th/0608227.

70. M. R. Douglas and W. Taylor, "The landscape of intersecting brane models," JHEP 0701, 031 (2007) arXiv:hep-th/0606109.

71. F. Marchesano and G. Shiu, "Building MSSM flux vacua," JHEP 0411, 041 (2004) arXiv:hep-th/0409132.

72. F. Gmeiner, D. Lüst and M. Stein, "Statistics of intersecting D-brane models on $T^{6} / Z_{6}, "$ arXiv:hep-th/0703011.

73. G. Honecker and T. Ott, "Getting just the supersymmetric standard model at intersecting branes on the Z(6)-orientifold," Phys. Rev. D 70, 126010 (2004) [Erratum-ibid. D 71, 069902 (2005)] arXiv:hep-th/0404055.

74. F. Gmeiner and M. Stein, "Statistics of SU(5) D-brane models on a type II orientifold," arXiv:hep-th/0603019

75. R. Blumenhagen and T. Weigand, "Chiral supersymmetric Gepner model orientifolds," JHEP 0402, 041 (2004) arXiv:hep-th/0401148.

76. T. P. T. Dijkstra, L. R. Huiszoon and A. N. Schellekens, "Chiral supersymmetric standard model spectra from orientifolds of Gepner models," Phys. Lett. B 609, 408 (2005) arXiv:hep-th/0403196.

77. T. P. T. Dijkstra, L. R. Huiszoon and A. N. Schellekens, "Supersymmetric standard model spectra from RCFT orientifolds," Nucl. Phys. B 710, 3 (2005) arXiv:hep-th/0411129.

78. P. Anastasopoulos, T. P. T. Dijkstra, E. Kiritsis and A. N. Schellekens, "Orientifolds, hypercharge embeddings and the standard model," Nucl. Phys. B 759, 83 (2006) arXiv:hep-th/0605226.

79. L. E. Ibanez, A. N. Schellekens and A. M. Uranga, "Instanton Induced Neutrino Majorana Masses in CFT Orientifolds with MSSM-like spectra," arXiv:0704.1079 [hepth].

80. K. R. Dienes and M. Lennek, "Fighting the floating correlations: Expectations and complications in extracting statistical correlations from the string theory landscape," Phys. Rev. D 75, 026008 (2007) arXiv:hep-th/0610319.

81. M. Grana, "Flux compactifications and generalized geometries," Class. Quant. Grav. 23, S883 (2006).

82. F. Denef, M. R. Douglas and S. Kachru, "Physics of string flux compactifications," arXiv:hep-th/0701050

83. S. Ashok and M. R. Douglas, "Counting flux vacua," JHEP 0401 (2004) 060 arXiv:hep-th/0307049.

84. M. Dine, E. Gorbatov and S. D. Thomas, "Low energy supersymmetry from the landscape," arXiv:hep-th/0407043

85. F. Denef and M. R. Douglas, "Distributions of nonsupersymmetric flux vacua," JHEP 0503, 061 (2005) arXiv:hep-th/0411183.

86. N. Arkani-Hamed and S. Dimopoulos, "Supersymmetric unification without low energy supersymmetry and signatures JHEP 0506, 073 (2005) arXiv:hep-th/0405159.

87. N. Arkani-Hamed, S. Dimopoulos and S. Kachru, "Predictive landscapes and new physics at a TeV," arXiv:hep-th/0501082

88. S. Gukov, C. Vafa and E. Witten, "CFT's from Calabi-Yau four-folds," Nucl. Phys. B 584 (2000) 69 [Erratum-ibid. B 608 (2001) 477] arXiv:hep-th/9906070.

89. T. R. Taylor and C. Vafa, "RR flux on Calabi-Yau and partial supersymmetry breaking," Phys. Lett. B 474 (2000) 130 arXiv:hep-th/9912152.

90. S. B. Giddings, S. Kachru and J. Polchinski, "Hierarchies from fluxes in string compactifications," Phys. Rev. D 66 (2002) 106006 arXiv:hep-th/0105097.

91. S. Kachru, R. Kallosh, A. Linde and S. P. Trivedi, "De Sitter vacua in string theory," 
Phys. Rev. D 68 (2003) 046005 arXiv:hep-th/0301240.

92. E. Witten, "Non-Perturbative Superpotentials In String Theory," Nucl. Phys. B 474 (1996) 343 arXiv:hep-th/9604030.

93. A. Achucarro, B. de Carlos, J. A. Casas and L. Doplicher, "de Sitter vacua from uplifting D-terms in effective supergravities from realistic strings," JHEP 0606, 014 (2006) arXiv:hep-th/0601190.

94. R. Blumenhagen, M. Cvetic and T. Weigand, "Spacetime instanton corrections in 4D string vacua - the seesaw mechanism for D-brane models," Nucl. Phys. B 771, 113 (2007) arXiv:hep-th/0609191.

95. M. Haack, D. Krefl, D. Lüst, A. Van Proeyen and M. Zagermann, "Gaugino condensates and D-terms from D7-branes," JHEP 0701, 078 (2007) arXiv:hep-th/0609211.

96. I. Affleck, M. Dine and N. Seiberg, "Supersymmetry Breaking By Instantons," Phys. Rev. Lett. 51, 1026 (1983).

97. T. R. Taylor, G. Veneziano and S. Yankielowicz, "Supersymmetric QCD And Its Massless Limit: An Effective Lagrangian Analysis," Nucl. Phys. B 218, 493 (1983).

98. O. J. Ganor, "On zeroes of superpotentials in F-theory," Nucl. Phys. Proc. Suppl. 67, 25 (1998).

99. D. Lüst and S. Stieberger, "Gauge threshold corrections in intersecting brane world models," arXiv:hep-th/0302221

100. N. Akerblom, R. Blumenhagen, D. Lüst and M. Schmidt-Sommerfeld, "Thresholds for intersecting D-branes revisited," arXiv:0705.2150 [hep-th].

101. D. Lüst, P. Mayr, R. Richter and S. Stieberger, "Scattering of gauge, matter, and moduli fields from intersecting branes," Nucl. Phys. B 696, 205 (2004) arXiv:hep-th/0404134.

102. N. Akerblom, R. Blumenhagen, D. Lüst and M. Schmidt-Sommerfeld, "Instantons and Holomorphic Couplings in Intersecting D-brane Models," arXiv:0705.2366 [hepth].

103. L. Görlich, S. Kachru, P. K. Tripathy and S. P. Trivedi, "Gaugino condensation and nonperturbative superpotentials in flux compactifications," JHEP 0412 (2004) 074 arXiv:hep-th/0407130.

104. P. K. Tripathy and S. P. Trivedi, "D3 brane action and fermion zero modes in presence of background flux," JHEP 0506 (2005) 066 arXiv:hep-th/0503072.

105. E. Bergshoeff, R. Kallosh, A. K. Kashani-Poor, D. Sorokin and A. Tomasiello, "An index for the Dirac operator on D3 branes with background fluxes," JHEP 0510 (2005) 102 arXiv:hep-th/0507069.

106. D. Lüst, S. Reffert, W. Schulgin and P. K. Tripathy, "Fermion zero modes in the presence of fluxes and a non-perturbative superpotential," JHEP 0608, 071 (2006) arXiv:hep-th/0509082.

107. L. E. Ibanez and A. M. Uranga, "Neutrino Majorana masses from string theory instanton effects," JHEP 0703, 052 (2007) arXiv:hep-th/0609213.

108. B. Florea, S. Kachru, J. McGreevy and N. Saulina, "Stringy instantons and quiver gauge theories," arXiv:hep-th/0610003.

109. N. Akerblom, R. Blumenhagen, D. Lüst, E. Plauschinn and M. Schmidt-Sommerfeld, "Non-perturbative SQCD Superpotentials from String Instantons," JHEP 0704, 076 (2007) arXiv:hep-th/0612132.

110. M. Bianchi and E. Kiritsis, "Non-perturbative and Flux superpotentials for Type I strings on the $Z_{3}$ orbifold," arXiv:hep-th/0702015.

111. M. Cvetic, R. Richter and T. Weigand, "Computation of D-brane instanton induced superpotential couplings - Majorana masses from string theory," arXiv:hep-th/0703028 
112. R. Argurio, M. Bertolini, G. Ferretti, A. Lerda and C. Petersson, "Stringy Instantons at Orbifold Singularities," arXiv:0704.0262 [hep-th].

113. M. Bianchi, F. Fucito and J. F. Morales, "D-brane Instantons on the $T^{6} / Z_{3}$ orientifold," arXiv:0704.0784 [hep-th].

114. S. Antusch, L. E. Ibanez and T. Macri, "Neutrino Masses and Mixings from String Theory Instantons," arXiv:0706.2132 [hep-ph].

115. R. Blumenhagen, M. Cvetic, D. Lüst, R. Richter and T. Weigand, "Non-perturbative Yukawa couplings from String Instantons," arXiv:0707.1871 [hep-th].

116. K. Choi, A. Falkowski, H. P. Nilles, M. Olechowski and S. Pokorski, "Stability of flux compactifications and the pattern of supersymmetry breaking," JHEP 0411 (2004) 076 arXiv:hep-th/0411066.

117. D. Lüst, S. Reffert, W. Schulgin and S. Stieberger, "Moduli stabilization in type IIB orientifolds. I: Orbifold limits," Nucl. Phys. B 766, 68 (2007) arXiv:hep-th/0506090.

118. J. J. Blanco-Pillado, R. Kallosh and A. Linde, "Supersymmetry and stability of flux vacua," arXiv:hep-th/0511042.

119. D. Krefl and D. Lüst, "On supersymmetric Minkowski vacua in IIB orientifolds," arXiv:hep-th/0603166

120. F. Denef, M. R. Douglas, B. Florea, A. Grassi and S. Kachru, "Fixing all moduli in a simple F-theory compactification," Adv. Theor. Math. Phys. 9, 861 (2005) arXiv:hep-th/0503124.

121. S. Reffert and E. Scheidegger, "Moduli stabilization in toroidal type IIB orientifolds," Fortsch. Phys. 54, 462 (2006) arXiv:hep-th/0512287.

122. D. Lüst, S. Reffert, E. Scheidegger, W. Schulgin and S. Stieberger, "Moduli stabilization in type IIB orientifolds. II," Nucl. Phys. B 766, 178 (2007) arXiv:hep-th/0609013.

123. D. Lüst, S. Reffert, E. Scheidegger and S. Stieberger, "Resolved toroidal orbifolds and their orientifolds," arXiv:hep-th/0609014.

124. S. Reffert, "Toroidal orbifolds: Resolutions, orientifolds and applications in string phenomenology," arXiv:hep-th/0609040

125. S. Reffert, "The Geometer's Toolkit to String Compactifications," arXiv:0706.1310 [hep-th].

126. C. P. Burgess, R. Kallosh and F. Quevedo, "de Sitter string vacua from supersymmetric D-terms," JHEP 0310, 056 (2003) arXiv:hep-th/0309187.

127. H. Jockers and J. Louis, "D-terms and F-terms from D7-brane fluxes," Nucl. Phys. B 718, 203 (2005) arXiv:hep-th/0502059.

128. G. Villadoro and F. Zwirner, "de Sitter vacua via consistent D-terms," Phys. Rev. Lett. 95, 231602 (2005) arXiv:hep-th/0508167.

129. E. Dudas and Y. Mambrini, "Moduli stabilization with positive vacuum energy," JHEP 0610, 044 (2006) arXiv:hep-th/0607077.

130. E. Dudas, C. Papineau and S. Pokorski, "Moduli stabilization and uplifting with dynamically generated F-terms," JHEP 0702, 028 (2007) arXiv:hep-th/0610297.

131. O. Lebedev, V. Lowen, Y. Mambrini, H. P. Nilles and M. Ratz, "Metastable vacua in flux compactifications and their phenomenology," JHEP 0702, 063 (2007) arXiv:hep-ph/0612035.

132. K. Intriligator, N. Seiberg and D. Shih, "Dynamical SUSY breaking in meta-stable vacua," JHEP 0604, 021 (2006) arXiv:hep-th/0602239.

133. S. Franco and A. M. .. Uranga, "Dynamical SUSY breaking at meta-stable minima from D-branes at obstructed geometries," JHEP 0606, 031 (2006) arXiv:hep-th/0604136.

134. H. Ooguri and Y. Ookouchi, "Meta-stable supersymmetry breaking vacua on inter- 
secting branes," Phys. Lett. B 641, 323 (2006) arXiv:hep-th/0607183.

135. P. G. Camara, L. E. Ibanez and A. M. Uranga, "Flux-induced SUSY-breaking soft terms," Nucl. Phys. B 689, 195 (2004) arXiv:hep-th/0311241.

136. M. Grana, T. W. Grimm, H. Jockers and J. Louis, "Soft supersymmetry breaking in Calabi-Yau orientifolds with D-branes and fluxes," Nucl. Phys. B 690, 21 (2004) arXiv:hep-th/0312232.

137. D. Lüst, S. Reffert and S. Stieberger, "Flux-induced soft supersymmetry breaking in chiral type IIB orientifolds with D3/D7-branes," Nucl. Phys. B 706, 3 (2005) arXiv:hep-th/0406092.

138. P. G. Camara, L. E. Ibanez and A. M. Uranga, "Flux-induced SUSYbreaking soft terms on D7-D3 brane systems," Nucl. Phys. B 708, 268 (2005) arXiv:hep-th/0408036.

139. D. Lüst, S. Reffert and S. Stieberger, "MSSM with soft SUSY breaking terms from D7-branes with fluxes," Nucl. Phys. B 727, 264 (2005) arXiv:hep-th/0410074.

140. A. Font and L. E. Ibanez, "SUSY-breaking soft terms in a MSSM magnetized D7brane model," JHEP 0503, 040 (2005) arXiv:hep-th/0412150.

141. D. Lüst, P. Mayr, S. Reffert and S. Stieberger, "F-theory flux, destabilization of orientifolds and soft terms on D7-branes," Nucl. Phys. B 732, 243 (2006) arXiv:hep-th/0501139.

142. K. Choi, A. Falkowski, H. P. Nilles and M. Olechowski, "Soft supersymmetry breaking in KKLT flux compactification," Nucl. Phys. B 718, 113 (2005) arXiv:hep-th/0503216.

143. E. Dudas and S. K. Vempati, "Large D-terms, hierarchical soft spectra and moduli stabilisation," Nucl. Phys. B 727, 139 (2005) arXiv:hep-th/0506172.

144. J. P. Conlon, S. S. Abdussalam, F. Quevedo and K. Suruliz, "Soft SUSY breaking terms for chiral matter in IIB string compactifications," JHEP 0701, 032 (2007) arXiv:hep-th/0610129.

145. J. P. Conlon, C. H. Kom, K. Suruliz, B. C. Allanach and F. Quevedo, "Sparticle Spectra and LHC Signatures for Large Volume String Compactifications," arXiv:0704.3403 [hep-ph].

146. K. Behrndt, G. Lopes Cardoso and D. Lüst, "Curved BPS domain wall solutions in four-dimensional N = 2 supergravity," Nucl. Phys. B 607, 391 (2001) arXiv:hep-th/0102128.

147. R. Kallosh, "Flux vacua as supersymmetric attractors," arXiv:hep-th/0509112.

148. H. Ooguri, C. Vafa and E. P. Verlinde, "Hartle-Hawking wave-function for flux compactifications," Lett. Math. Phys. 74 (2005) 311 arXiv:hep-th/0502211.

149. S. Gukov, K. Saraikin and C. Vafa, "The entropic principle and asymptotic freedom," Phys. Rev. D 73, 066010 (2006) arXiv:hep-th/0509109.

150. B. Fiol, "On the critical points of the entropic principle," JHEP 0603, 054 (2006) arXiv:hep-th/0602103.

151. G. L. Cardoso, D. Lüst and J. Perz, "Entropy maximization in the presence of highercurvature interactions," JHEP 0605, 028 (2006) arXiv:hep-th/0603211.

152. R. Gopakumar and C. Vafa, "M-theory and topological strings. I," arXiv:hep-th/9809187.

153. G. L. Cardoso, V. Grass, D. Lüst and J. Perz, "Extremal non-BPS black holes and entropy extremization," JHEP 0609, 078 (2006) arXiv:hep-th/0607202.

154. J. P. Derendinger, C. Kounnas, P. M. Petropoulos and F. Zwirner, "Superpotentials in IIA compactifications with general fluxes," Nucl. Phys. B 715, 211 (2005) arXiv:hep-th/0411276.

155. G. Villadoro and F. Zwirner, "N = 1 effective potential from dual type-IIA D6/O6 
orientifolds with general fluxes," JHEP 0506, 047 (2005) arXiv:hep-th/0503169.

156. O. DeWolfe, A. Giryavets, S. Kachru and W. Taylor, "Type IIA moduli stabilization," JHEP 0507, 066 (2005) arXiv:hep-th/0505160.

157. P. G. Camara, A. Font and L. E. Ibanez, "Fluxes, moduli fixing and MSSM-like vacua in a simple IIA orientifold," JHEP 0509, 013 (2005) arXiv:hep-th/0506066.

158. D. Lüst and D. Tsimpis, "Supersymmetric AdS(4) compactifications of IIA supergravity," JHEP 0502, 027 (2005) arXiv:hep-th/0412250.

159. G. Villadoro and F. Zwirner, "Beyond Twisted Tori," arXiv:0706.3049 [hep-th].

160. K. Becker, M. Becker, C. Vafa and J. Walcher, Nucl. Phys. B 770, 1 (2007) arXiv:hep-th/0611001.

161. K. Becker, M. Becker and J. Walcher, arXiv:0706.0514 [hep-th].

162. C. Kounnas, D. Lüst, M. Petropoulos and D. Tsimpis, " $A d S_{4}$ flux vacua in type II superstrings and their domain wall solutions," to appear.

163. H. Ooguri and C. Vafa, "On the geometry of the string landscape and the swampland," Nucl. Phys. B 766, 21 (2007) arXiv:hep-th/0605264. 